Nervenarzt 2015 $\cdot 86: 923-924$

DOI 10.1007/s00115-014-4247-8

Online publiziert: 26. Juli 2015

๑) Springer-Verlag Berlin Heidelberg 2015

\author{
H.-P. Hartung ${ }^{1} \cdot$ R. Gold ${ }^{2}$ \\ ${ }^{1}$ Neurologische Klinik, Medizinische Fakultät, Heinrich-Heine-Universität Düsseldorf \\ ${ }^{2}$ Klinik für Neurologie, Katholisches Klinikum Bochum, St. Josef-Hospital, \\ Universitätsklinikum der Ruhr-Universität Bochum
}

\title{
Mensch im Blick, Gehirn im Fokus
}

Umweltfaktoren und Lifestyle bei wichtigen Erkrankungen aus unserem Fachgebiet beleuchten. Auf den ersten Blick vermag dies etwas unwissenschaftlich anmuten, werden doch unsere wissenschaftlichen Methoden immer genauer, detaillierter und komplexer. Aber gerade in einem Zeitalter evidenzbasierter Medizin sind solche Fragen nur unter streng definierten Bedingungen an ausreichend großen Kohorten zu untersuchen. Der Frage, ob und in welchem Umfang Lebensstilfaktoren das Risiko beeinflussen, an Alzheimer oder Parkinson zu erkranken, gehen J.B. Schulz aus Aachen und G. Deuschl aus Kiel im Beitrag „Einfluss des Lebensstils auf neurodegenerative Erkrankungen" nach. Gerade für diese zwei genannten neurodegenerativen Erkrankungen liegen überzeugende Daten vor, die einen protektiven Effekt bestimmter Lebensstilfaktoren unterstreichen. Die Frage, ob Lifestyle und/oder Umweltfaktoren einen Einfluss auf eine klinisch manifeste neurogenerative Erkrankung haben, bleibt derzeit weiterhin ungelöst. L.M. Gerischer, A. Flöel und M. Endres aus Berlin widmen sich derselben Frage mit dem Thema „Schlaganfall - Lifestyle und Umwelt“. Für die Schlaganfallprävention sind die Effekte von körperlicher Aktivität, Körpergewicht, Ernährung, Rauchen, Stress bis hin zum Haustier ausführlich untersucht und diskutiert. Welchen Effekt Umweltfaktoren auf die Rehabilitation haben, wird derzeit intensiv untersucht, erste positive Hinweise unterstützen Forschungsinitiativen auf diesem wichtigen Forschungsgebiet. Bei der Multiplen Skle- rose findet neben Umweltfaktoren wie Rauchen und Vitamin-D-Mangel als Risikofaktoren neuerdings die mögliche Rolle der individuellen Darmflora besonderes Interesse. Der Zusammenhang ist von $R$. Hohlfeld und $H$. Wekerle unter dem Titel „Multiple Sklerose und Mikrobiota vom Genom zum Metagenom?" zusammengefasst worden. Ein besseres Verständnis der Interaktionen zwischen dem darmassoziierten Immungewebe und einer systemischen Immunreaktion hätte nicht nur Implikationen für die Multiple Sklerose, sondern weitreichende Folgen für das Verständnis der meisten immunvermittelten chronischen Erkrankungen.

Der Multiplen Sklerose ist auch das Präsidentensymposium auf dem Kongress gewidmet -entsprechend den Schwerpunkten der Düsseldorfer und Bochumer Kliniken. Auch in diesem Heft fasst eine Arbeitsgruppe aus Düsseldorf und Hannover (D. Kremer, H.-P. Hartung, M. Stangel und P. Küry) „Neue Therapiestrategien zur Myelinreparatur bei der Multiplen Sklerose“ zusammen. Aus Rostock und Düsseldorf (A. Winkelmann, $M$. Löbermann, E.C. Reisinger, H.-P. Hartung, U.K. Zettl) stammen zwei Beiträge zu infektiologischen Risiken in der MultipleSklerose-Therapie durch Immuntherapeutika.

In all diesen Feldern sind in jüngster Zeit spannende Zusammenhänge erforscht worden. Diese leisten nicht nur einen Beitrag zum besseren Verständnis von Ursachen und pathogenetischen $\mathrm{Zu}$ sammenhängen, sondern zeigen auch innovative Möglichkeiten der Prävention
Gehirn im Fokus“ die Bedeutung von 
und Therapie auf. Weitere spannende Ergebnisse sind auf dem Kongress zu erwarten und zu diskutieren.

Wir freuen uns auf einen anregenden Wissens- und Erfahrungsaustausch in Düsseldorf.

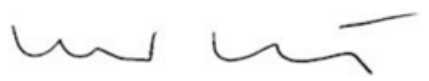

Prof. Dr. Hans-Peter Hartung

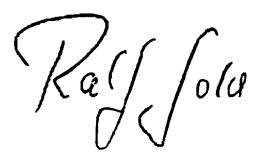

Prof. Dr. Ralf Gold

Mit Ihren Kongresssekretären Prof. Dr. Bernd C. Kieseier und Prof. Dr. Aiden Haghikia

\section{Korrespondenzadressen}

\section{Prof. Dr. H.-P. Hartung}

Neurologische Klinik, Medizinische Fakultät, Heinrich-Heine-Universität Düsseldorf Moorenstr. 5, 40225 Düsseldorf hans-peter.hartung@uni-duesseldorf.de

\section{Prof. Dr. R. Gold}

Klinik für Neurologie, Katholisches Klinikum Bochum, St. Josef-Hospital, Universitätsklinikum der Ruhr-Universität Bochum Gudrunstr. 56, 44791 Bochum ralf.gold@rub.de

Interessenkonflikt. H.-P. Hartung und R. Gold geben an, dass kein Interessenkonflikt besteht.

\section{Ihr Zugang zum Onlinearchiv}

Im Volltextarchiv auf springermedizin.de stehen Ihnen alle elektronisch verfügbaren Ausgaben Ihrer Zeitschrift zur Verfügung - unabhängig davon, seit wann Sie die Zeitschrift abonniert haben. Die Beiträge sind als PDF-Datei im Layout der gedruckten Ausgabe und als HTML-Version verfügbar. In der HTML-Version ist die Literatur verlinkt, sodass Sie direkt zu den zitierten Quellen gelangen können.

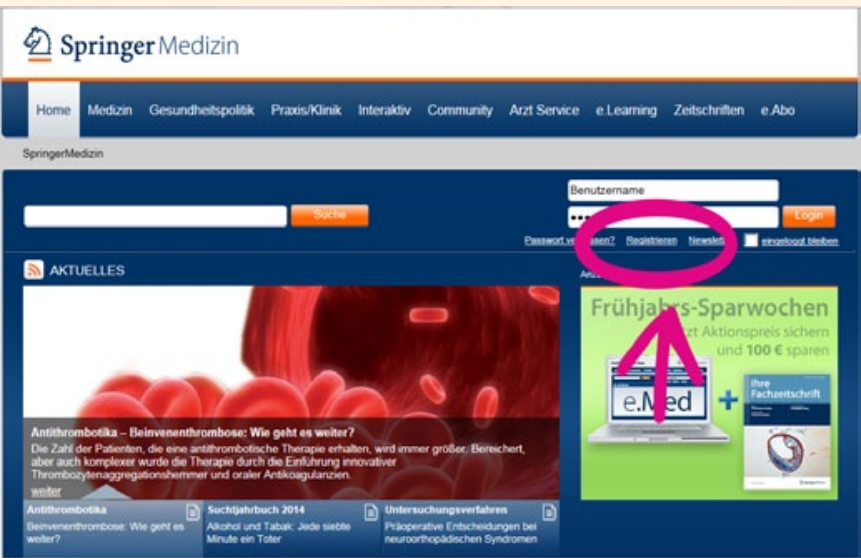

\section{Und so einfach geht es:}

\section{Einmalig registrieren:}

Registrieren Sie sich auf www.springermedizin.de/der-nervenarzt über den Menüpunkt Registrieren (siehe Abbildung oben). Geben Sie dabei Ihre Abonummer an und ggf. die Adresse, an die Ihre Zeitschrift geliefert wird, sowie Ihre EFN-Nummer.

\section{Log in:}

Nach erfolgreicher Registrierung werden Ihnen Ihre Zugangsdaten per E-Mail zugeschickt.

\section{Ausgabenarchiv:}

Im Ausgabenarchiv unter www.springermedizin.de/der-nervenarzt finden Sie alle elektronisch verfügbaren Ausgaben der Zeitschrift, sortiert nach Jahrgang und Ausgabennummer. Die einzelnen Beiträge werden im Inhaltsverzeichnis unter Angabe von Rubrik, Titel und Autoren übersichtlich angezeigt. Die Reihenfolge entspricht der im gedruckten Heft. Mit einem Klick gelangen Sie direkt zum gewünschten Beitrag.

Für alle Fragen zu Ihrem Onlinezugang steht Ihnen unser Kundenservice unter Kundenservice@springermedizin.de zur Verfügung. Telefonisch erreichen Sie die Hotline montags bis freitags von $9.00 \mathrm{bis} 17.00 \mathrm{Uhr}$ kostenfrei unter 0800-77 80777 sowie gebührenpflichtig aus dem Ausland unter +4930884293600. 